\title{
First reported foodborne outbreak associated with microsporidia, Sweden, October 2009
}

\author{
V. DECRAENE ${ }^{1,2 *}$, M. LEBBAD ${ }^{3}$, S. BOTERO-KLEIVEN ${ }^{3}$, A.-M. GUSTAVSSON ${ }^{4}$ \\ AND M. LÖFDAHL ${ }^{5}$ \\ ${ }^{1}$ Department of Analysis and Prevention, Swedish Institute for Communicable Disease Control, Solna, Sweden \\ ${ }^{2}$ European Programme for Intervention Epidemiology Training (EPIET), European Centre for Disease \\ Prevention and Control, Stockholm, Sweden \\ ${ }^{3}$ Department of Diagnostics and Vaccinology, Swedish Institute for Communicable Disease Control, Solna, \\ Sweden \\ ${ }^{4}$ Värmland County Council, Karlstad, Sweden \\ ${ }^{5}$ Department of Preparedness, Swedish Institute for Communicable Disease Control, Solna, Sweden
}

(Accepted 23 March 2011; first published online 9 May 2011)

\section{SUMMARY}

Microsporidia are spore-forming intracellular parasites that infrequently cause disease in immunocompetent persons. This study describes the first report of a foodborne microsporidiosis outbreak which affected persons visiting a hotel in Sweden. Enterocytozoon bieneusi was identified in stool samples from 7/11 case-patients, all six sequenced samples were genotype $\mathrm{C}$. To confirm that this was not a chance finding, 19 stool samples submitted by healthy persons from a comparable group who did not visit the hotel on that day were tested; all were negative for microsporidia. A retrospective cohort study identified 135 case-patients (attack rate $30 \%$ ). The median incubation period was 9 days. Consumption of cheese sandwiches [relative risk (RR) $4 \cdot 1$,

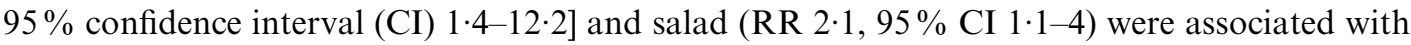
illness. Both items contained pre-washed, ready-to-eat cucumber slices. Microsporidia may be an under-reported cause of gastrointestinal outbreaks; we recommend that microsporidia be explored as potential causative agents in food- and waterborne outbreaks, especially when no other organisms are identified.

Key words: Cohort study, emerging, Enterocytozoon bieneusi, foodborne, microsporidia, outbreak, Sweden.

\section{INTRODUCTION}

Microsporidia are single-celled, spore-forming obligate intracellular parasites which, prior to 1985, were only sporadically reported in humans. They have since emerged as a major cause of opportunistic infections associated with persistent diarrhoea and weight loss in

\footnotetext{
* Author for correspondence: Dr V. Decraene, Swedish Institute for Communicable Disease Control/Smittskyddsinstitutet, Nobels väg 18, 17182 Solna, Sweden.

(Email: valerie.decraene@smi.se)
}

persons with AIDS [1] and organ transplant patients [2-4]. While still uncommonly reported in immunocompetent persons, intestinal microsporidiosis is increasingly being identified in children, the elderly, and travellers [5-8]. In healthy persons, disease is characterized by self-limiting diarrhoea usually of $\leqslant 1$ month's duration [1]. The incubation period for microsporidiosis remains unknown.

Although there are more than 1200 species of microsporidia, most infections in humans are caused by two species: Enterocytozoon (E.) bieneusi and

The online version of this article is published within an Open Access environment subject to the conditions of the Creative Commons Attribution-NonCommercial-ShareAlike licence $<$ http://creativecommons.org/licenses/by-nc-sa/2.5/ $>$. The written permission of Cambridge University Press must be obtained for commercial re-use. 
Encephalitozoon intestinalis [9]. E. bieneusi has been identified in both immunocompromised and healthy persons and has also been isolated from a wide range of animal hosts including cattle, pigs and a variety of birds $[6,10,11]$. More than 90 genotypes of E. bieneusi are recognized, some of which are associated with a single host and others with $\geqslant 2$ hosts [12]. Possible modes of transmission include person-to-person, zoonotic transmission, inhalation of contaminated aerosols and ingestion of contaminated food and water [6]. Only one outbreak of microsporidiosis, associated with waterborne transmission, has been reported in the literature [13].

Previously, microsporidia research in Sweden had focused on HIV-positive patients [14, 15]. In contrast, only one case of $E$. bieneusi infection in an immunocompetent person has been reported [16]. The case-patient was an HIV-negative man who had not travelled abroad in the previous 12 months and was identified as part of a 1-year prospective study to identify enteropathogens in adults with diarrhoea and healthy control subjects [17]. Of 417 patients with diarrhoea found to be shedding enteropathogens, only this one microsporidiosis case was identified; out of 203 controls none were found to have microsporidia.

We describe a large foodborne outbreak of microsporidiosis associated with a hotel in central Sweden. On 5 November 2009, the regional public health authority in Värmland County was informed of more than 100 cases of gastrointestinal illness in 471 attendees of a 1-day conference held at a hotel on 23 October 2009 (group A). A second group of visitors $(n=15$, group B) attended a separate meeting at the hotel on the same day but reported no illness. Staff members working on 23 October $(n=39)$ had also eaten the same foods on that day and two reported illness after the event. In collaboration with the regional public health and environmental health authorities, the Swedish Institute for Communicable Disease Control (SMI) initiated an investigation in order to determine the mode and vehicle of transmission, establish the magnitude of the outbreak, and identify the aetiological agent.

\section{METHODS}

\section{Laboratory investigations}

Outbreak samples

All persons who reported illness were asked to submit a stool sample. The county microbiology laboratory investigated 95 samples for bacterial enteropathogens (Salmonella, Shigella, Campylobacter, enterohaemorrhagic Escherichia coli, Vibrio, Plesiomonas, Aeromonas) and intestinal parasites (Entamoeba spp., Giardia, Isospora, Cyclospora, Cryptosporidium).

Of these initial stool samples, only eight (formalinfixed) samples remained for further testing and were sent to SMI's parasitology reference laboratory where they were investigated by light microscopy for Entamoeba spp., Dientamoeba, Giardia, Cryptosporidium, Blastocystis and Cyclospora. Subsequent examination for the presence of microsporidian spores was performed by light microscopy after modified trichrome staining [18] and monoclonal antibody immunofluorescence assay (Bordier Affinity Products SA, Switzerland). DNA was extracted directly from stool specimens by using the QIAamp DNA mini kit (Qiagen, Germany) after an initial disruption of the spores with a Mini-BeadBeater (Biospec Products Inc., USA). Amplification of the ITS region was performed by using the primers for nested PCR described by Buckholt et al. [19]. Amplicons from all PCR-positive samples were directly sequenced in both directions. The BLAST tool was used to compare the nucleotide sequences with sequences in the GenBank database.

A further three samples from persons still experiencing symptoms in late November were analysed for the presence of norovirus, rotavirus and adenovirus. These samples were subsequently sent to SMI's virology laboratory where they were tested for astrovirus, rotavirus, adenovirus, norovirus and sapovirus. They were also later investigated for microsporidia at SMI's parasitology laboratory.

DNA from all samples available at SMI $(n=11)$ were sent to the reference parasitology laboratory at Statens Serum Institut (SSI) in Copenhagen for investigation of E. bieneusi and Encephalitozoon spp. by real-time PCR [20].

\section{Prevalence study samples}

In June 2010, all members of the same professional group as group A who had not attended the conference on 23 October $(n \approx 70)$ were contacted and invited to participate in a prevalence study. They were asked to provide a stool sample and complete a short paper questionnaire regarding sex, age, travel history during the previous 6 months, and gastrointestinal illness during the previous 4 weeks. DNA from samples was extracted and amplified as described above. Ethical approval was obtained from 
the ethical review board at Karolinska Institute, Solna, Sweden.

\section{Epidemiological investigation}

We conducted a retrospective cohort study which included the three identifiable groups who ate at the hotel on 23 October: group A, group B and the hotel staff who worked on that day. All participants were asked to complete a web-based questionnaire (Artisan Global Software, Sweden; www.artologik.com). For group A and the hotel staff, the link to the questionnaire was sent by email on 18 November. For group B, only postal addresses were available so all 15 persons were sent a letter with the link to the questionnaire together with a paper copy. The questionnaire asked about date and time of illness onset, type and duration of symptoms, foods consumed during the entire day (coffee breaks, lunch, dinner) and other activities at the hotel (overnight stay, use of spa facilities).

A case-patient was defined as a person who ate at the hotel on 23 October 2009, and subsequently developed at least one of the following symptoms: diarrhoea, vomiting, abdominal pain or nausea. An onset date was also required.

We analysed the data using Stata v. 10 (StataCorp, USA). Food-specific attack rates (AR), relative risks (RR) and 95\% confidence intervals (CIs) were calculated for all exposures. A Fisher's exact $P$ value of $<0.05$ was considered significant. Respondents who answered 'do not know' were excluded from the analysis of that particular exposure. For those persons who stated that they ate each meal and answered yes to one or more of the food items for that meal and who left all other answers blank, the missing values were coded as 'no'. For all others, missing values were retained and these persons were excluded from the analysis. The univariate analyses of the individual food items served during the morning coffee break and lunchtime meals were restricted to persons who attended those meals. To control for confounding, we performed logistic regression. For both the morning coffee break and lunchtime meals, food items that were significantly associated with illness $(P$ was $<0.05)$ and were considered to be biologically plausible sources of infection were fitted into a model together with sex and age group.

\section{Environmental investigation}

The local Environmental Health Officer (EHO) inspected the hotel kitchen on several occasions and interviewed the staff. In addition to evaluating the temperature controls and observing general hygiene practices, the EHO investigated the food handling and storage procedures for the foods served on 23 October. No leftover food samples were available for microbiological testing.

\section{RESULTS}

\section{Laboratory investigations}

\section{Outbreak samples}

Initially, 95 stool samples were obtained. All samples were negative for enteropathogenic bacteria and intestinal parasites at the county laboratory. The three additional samples from patients with continuing symptoms were negative for norovirus, rotavirus and adenovirus (Table 1).

None of the eight stool samples sent to SMI were positive for Giardia, Cryptosporidium, Cyclospora, Dientamoeba, Entamoeba spp., or Blastocystis. Similarly, the faecal samples from the three persons with persisting symptoms in late November were all negative for enteropathogenic viruses at SMI.

On 17 December, 3/8 initial samples received at SMI were found to contain microsporidia spores by microscopy. An immunofluorescence assay using monoclonal antibody identified the species in all three samples as E. bieneusi. This was further confirmed by PCR. Two of the three samples from late November were positive for E. bieneusi by microscopy, immunofluorescent staining and PCR; the remaining sample was positive only by PCR. Thus, a total of six samples were positive for E. bieneusi. Sequencing of these PCR-positive samples revealed that all six had sequences corresponding to the previously described genotype C, GenBank accession no. AF101199 (Table 1).

Real-time PCR performed by SSI detected E. bieneusi in 4/8 initial stool samples and all three of the samples from late November. This gave a total of $7 / 11$ positive samples. No samples were positive for Encephalitozoon spp.

\section{Prevalence study samples}

A total of 19 control group persons provided stool samples and completed the questionnaire. All samples were negative for microsporidia. The age and gender distribution of these persons was comparable to that of the outbreak cohort ( $95 \%$ female, median age 56 
Table 1. Summary of results from laboratory investigations of stool samples submitted following the hotel visit on 23 October 2009

\begin{tabular}{lr}
\hline \hline & $\begin{array}{c}\text { No. of positive } \\
\text { samples/no. of } \\
\text { samples tested }\end{array}$ \\
Laboratory results & $0 / 95$ \\
\hline Results from local laboratory & $0 / 95$ \\
$\quad$ Bacteria (Salmonella, Shigella, Campylobacter, enterohaemorrhagic Escherichia coli, & $0 / 3$ \\
$\quad$ Vibrio, Plesiomonas and Aeromonas) & \\
Parasites (Entamoeba spp., Isospora, Cyclospora, Giardia, Cryptosporidium) & $0 / 3$ \\
Viruses (norovirus, rotavirus, adenovirus) & $0 / 8$ \\
Results from SMI virology laboratory & $6 / 11 \dagger$ \\
$\quad$ Astrovirus, rotavirus, adenovirus, norovirus and sapovirus & \\
Results from SMI parasitology laboratory & \\
$\quad$ Giardia, Cryptosporidium, Cyclospora, Dientamoeba, Entamoeba sp., Blastocystis & $7 / 11 \dagger$ \\
Enterocytozooon bieneusi* & $0 / 11$ \\
Results from SSI parasitology laboratory & \\
E. bieneusi & \\
Encephalitozoon spp. & \\
\hline \hline
\end{tabular}

SMI, Swedish Institute for Communicable Disease Control, Solna, Sweden; SSI, Statens Serum Institut, Copenhagen, Denmark.

* E. bieneusi was detected by using light microscopy (chromotrope staining) in combination with an immunofluorescence assay.

$\dagger$ Of those samples positive for E. bieneusi at SMI, all six were genotype C: GenBank accession no. AF101199.

* SSI detected one further case by real-time PCR among the eight initial, formalin-fixed samples. This sample was not genotyped.

years) and none had experienced gastrointestinal illness in the previous 4 weeks.

\section{Epidemiological investigation}

In total, 450/525 (86\%) persons completed the questionnaire and 135 met the case definition (AR 30\%). Of the staff, only $3 / 39$ (AR $8 \%$ ) reported illness while in groups A and B the ARs were $33 \%(130 / 397)$ and $14 \%(2 / 14)$, respectively. The median age of the casepatients was 52 years (range 20-65 years) and most $(86 \%)$ were female; these figures were similar to those of the cohort as a whole (median age 52 years, $84 \%$ female).

Onset dates of the 135 case-patients ranged from 23 October to 13 November, with a peak on 2 November (Fig. 1). The distribution of case-patients over time indicates a point-source outbreak but is broad, reflecting a wide range of incubation periods. The median incubation time between the date of the conference and illness onset in case-patients was 9 days for all case-patients (range 0-21 days) and 7 days (range $3-15$ days) for the four microbiologically confirmed case-patients (Table 2).

The most common symptoms were abdominal pain $(87 \%)$, diarrhoea (82\%) and nausea (82\%) (Table 2).
Notably, vomiting was only present in $7 \%$ of casepatients. When asked about other symptoms (freetext field), 29 case-patients (21\%) reported feeling bloated, $14(10 \%)$ reported suffering from flatulence and $13(10 \%)$ reported experiencing fatigue. None of the case-patients had bloody diarrhoea. The median duration of illness was 6 days (range 1-20 days) although it should be noted that the regional public health authority was notified of case-patients who were still experiencing persistent or recurring symptoms as late as February 2010.

Persons who attended the morning coffee break were found to have a 6.4 times higher risk of illness than those who did not (Table 3). Lunch was also associated with illness (RR $5 \cdot 7,95 \%$ CI $0 \cdot 8-38 \cdot 1$, $P=0.018)$. Of the individual food items, the cheese sandwich served during the morning coffee break and the salad served during lunch had the highest RRs: $4 \cdot 1(95 \%$ CI $1 \cdot 4-12 \cdot 2, P=0 \cdot 001)$ and $2 \cdot 1(95 \% \mathrm{CI}$ $1 \cdot 1-4 \cdot 0, P=0 \cdot 013$ ), respectively (Table 3 ). Of the 135 cases, $130(97 \%)$ ate the cheese sandwich and 117 $(87 \%)$ ate the salad. Additionally, the mashed potatoes and bread were significantly associated with illness. Univariate analyses for food items served during the afternoon coffee break, at dinner, and for other snacks provided throughout the day were also 
Table 2. Summary of symptom distribution, symptom duration and incubation periods in case-patients during an outbreak of E. bieneusi, Sweden, October-November 2009

\begin{tabular}{|c|c|c|c|c|}
\hline & \multicolumn{2}{|c|}{ All case-patients $(n=135)$} & \multicolumn{2}{|c|}{ Confirmed case-patients $(n=4)^{*}$} \\
\hline & No. $(\%)$ & Median (range) & No. $(\%)$ & Median (range) \\
\hline \multicolumn{5}{|l|}{ Symptoms } \\
\hline Diarrhoea & $110(82)$ & & $4(100)$ & \\
\hline Abdominal pain & $117(87)$ & & $4(100)$ & \\
\hline Nausea & $111(82)$ & & $4(100)$ & \\
\hline Headache & $80(59)$ & & $3(75)$ & \\
\hline Muscle ache & $45(33)$ & & $2(50)$ & \\
\hline Fever $\left(>38^{\circ} \mathrm{C}\right)$ & $35(26)$ & & $3(75)$ & \\
\hline Bloating & $29(21)$ & & 0 & \\
\hline Flatulence $\dagger$ & $14(10)$ & & 0 & \\
\hline Fatigue $\dagger$ & $13(10)$ & & $2(50)$ & \\
\hline Vomiting & $10(7)$ & & 0 & \\
\hline Duration of symptoms (days) $\ddagger$ & & $6(1-20)$ & & n.a. $\ddagger$ \\
\hline Incubation period (days) & & $9(0-21) \S$ & & $7(3-15)$ \\
\hline
\end{tabular}

* Three of the seven microbiologically confirmed case-patients did not return the questionnaire and are therefore not included here.

$\dagger$ These symptoms were reported by case-patients in a free-text 'other symptoms' question.

$\$$ Median and range of symptom duration could only be calculated for those case-patients whose symptoms had ceased at the time of questionnaire completion and who did not have missing data $(n=89)$. For confirmed case-patients, three indicated that they were still ill so no median or range of symptom duration could be calculated.

$\S$ One case-patient developed symptoms on the same day as the event; the incubation time was 8-10 $\mathrm{h}$.

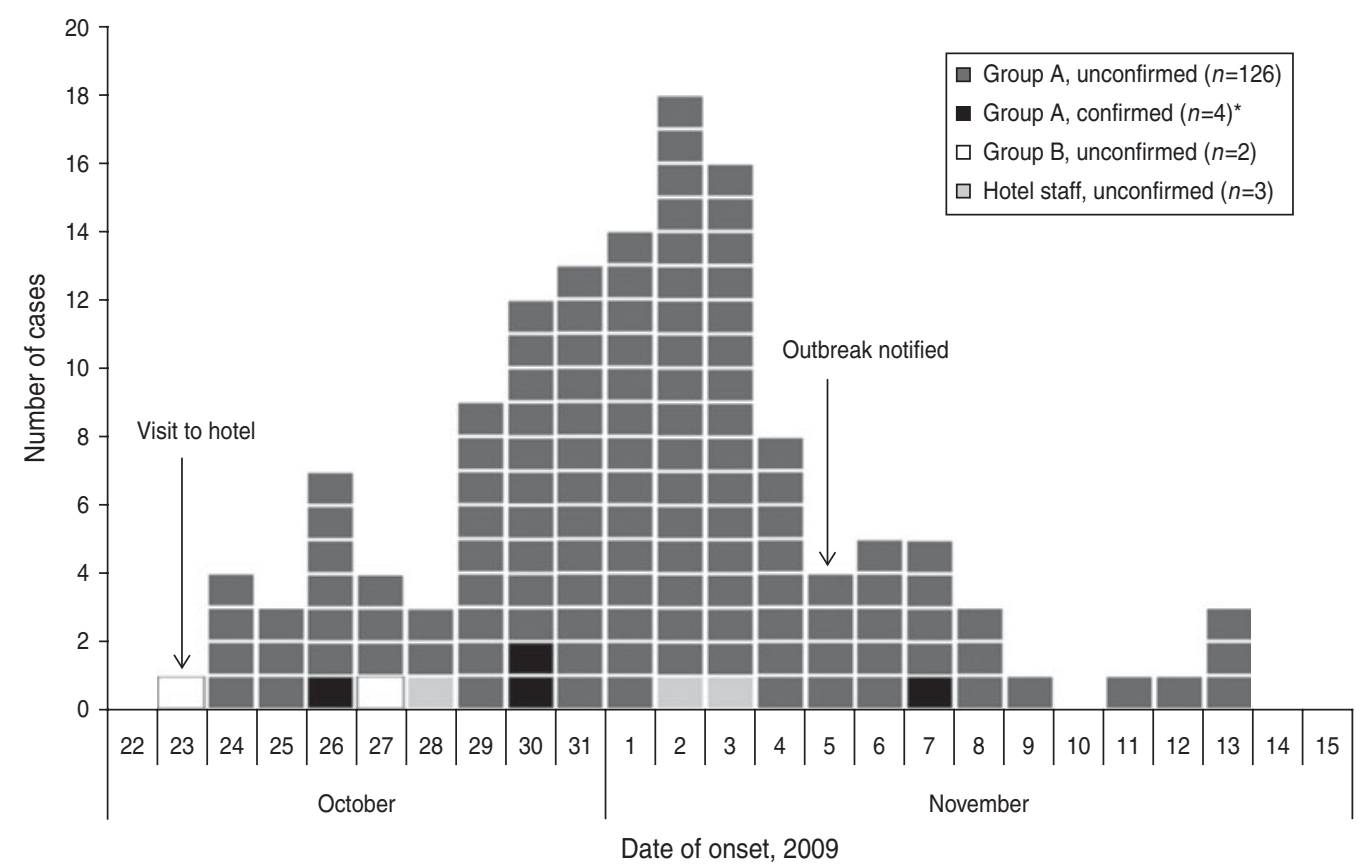

Fig. 1. Distribution of cases $(n=135)$ by date of symptom onset during an outbreak of E. bieneusi, Sweden, October-November 2009. * Three of the microbiologically confirmed case-patients did not return the questionnaire and are therefore not shown in the figure. Two of these three case-patients became ill on 2 November; an onset date for the remaining case-patient was not available. 
Table 3. Risk of illness by exposure to foods served during a coffee break and lunch on 23 October 2009, Sweden

\begin{tabular}{|c|c|c|c|c|c|c|c|c|c|c|}
\hline \multirow[b]{2}{*}{ Exposure } & \multicolumn{3}{|c|}{ Exposed } & \multicolumn{3}{|c|}{ Unexposed } & \multirow[b]{2}{*}{ RR } & \multirow[b]{2}{*}{$95 \% \mathrm{CI}$} & \multirow[b]{2}{*}{$P$ value* } & \multirow{2}{*}{$\begin{array}{l}\text { Cases } \\
\text { exposed } \\
(\%)\end{array}$} \\
\hline & Cases & Total & AR \% & Cases & Total & AR \% & & & & \\
\hline Morning coffee break & 133 & 407 & $32 \cdot 7$ & 2 & 39 & $5 \cdot 1$ & $6 \cdot 37$ & $1 \cdot 64-24 \cdot 76$ & $<0.000$ & $98 \cdot 5$ \\
\hline Cheese sandwich & 130 & 392 & $33 \cdot 2$ & 3 & 37 & $8 \cdot 1$ & $4 \cdot 09$ & $1 \cdot 37-12 \cdot 21$ & $0 \cdot 001$ & $96 \cdot 3$ \\
\hline Coffee & 108 & 322 & $33 \cdot 5$ & 25 & 106 & $23 \cdot 6$ & $1 \cdot 42$ & $0 \cdot 98-2 \cdot 07$ & $0 \cdot 069$ & $80 \cdot 0$ \\
\hline Tea & 15 & 56 & $26 \cdot 8$ & 118 & 375 & $31 \cdot 5$ & $0 \cdot 85$ & $0 \cdot 54-1 \cdot 35$ & $0 \cdot 537$ & $11 \cdot 1$ \\
\hline Milk & 47 & 123 & $38 \cdot 2$ & 86 & 305 & $28 \cdot 2$ & $1 \cdot 36$ & $1 \cdot 02-1 \cdot 81$ & $0 \cdot 050$ & $34 \cdot 8$ \\
\hline Dry cake & 17 & 41 & $41 \cdot 5$ & 115 & 382 & $30 \cdot 1$ & $1 \cdot 38$ & $0 \cdot 93-2 \cdot 04$ & $0 \cdot 156$ & $12 \cdot 6$ \\
\hline Fruit (grouped) & 21 & 84 & $25 \cdot 0$ & 113 & 349 & $32 \cdot 4$ & $0 \cdot 77$ & $0 \cdot 52-1 \cdot 15$ & $0 \cdot 237$ & $15 \cdot 6$ \\
\hline Lunch & 134 & 427 & $31 \cdot 4$ & 1 & 18 & $5 \cdot 6$ & $5 \cdot 65$ & $0 \cdot 84-38 \cdot 14$ & $0 \cdot 018$ & $99 \cdot 3$ \\
\hline Mashed potatoes & 128 & 388 & $33 \cdot 0$ & 6 & 38 & $15 \cdot 8$ & $2 \cdot 09$ & $0.99-4 \cdot 41$ & $0 \cdot 029$ & $94 \cdot 8$ \\
\hline Salmon & 129 & 407 & $31 \cdot 7$ & 5 & 25 & $20 \cdot 0$ & $1 \cdot 58$ & $0 \cdot 71-3 \cdot 52$ & $0 \cdot 270$ & $95 \cdot 6$ \\
\hline White wine sauce & 117 & 355 & $33 \cdot 0$ & 14 & 43 & $32 \cdot 6$ & $1 \cdot 01$ & $0 \cdot 64-1 \cdot 60$ & $1 \cdot 000$ & $86 \cdot 7$ \\
\hline Thyme & 99 & 291 & $34 \cdot 0$ & 24 & 85 & $28 \cdot 2$ & $1 \cdot 20$ & $0 \cdot 83-1 \cdot 75$ & $0 \cdot 359$ & $73 \cdot 3$ \\
\hline Salad $\dagger$ & 117 & 336 & $34 \cdot 8$ & 8 & 48 & $16 \cdot 7$ & $2 \cdot 09$ & $1 \cdot 09-4 \cdot 00$ & $0 \cdot 013$ & $86 \cdot 7$ \\
\hline Boiled vegetables & 95 & 300 & $31 \cdot 7$ & 28 & 83 & $33 \cdot 7$ & $0 \cdot 94$ & $0 \cdot 67-1 \cdot 32$ & $0 \cdot 791$ & $70 \cdot 4$ \\
\hline \multicolumn{11}{|l|}{ Vegetarian option } \\
\hline Quorn filet & 3 & 18 & $16 \cdot 7$ & 130 & 407 & $31 \cdot 9$ & $0 \cdot 52$ & $0 \cdot 18-1 \cdot 48$ & $0 \cdot 204$ & $2 \cdot 2$ \\
\hline Boiled potatoes & 13 & 47 & $27 \cdot 7$ & 118 & 371 & $31 \cdot 8$ & $0 \cdot 87$ & $0 \cdot 54-1 \cdot 41$ & $0 \cdot 620$ & $9 \cdot 6$ \\
\hline Grilled vegetables & 16 & 36 & $44 \cdot 4$ & 97 & 339 & $28 \cdot 6$ & $1 \cdot 55$ & $1 \cdot 04-2 \cdot 32$ & $0 \cdot 057$ & $11 \cdot 9$ \\
\hline Chilli sauce & 3 & 17 & $17 \cdot 7$ & 112 & 363 & $30 \cdot 9$ & $0 \cdot 57$ & $0 \cdot 20-1 \cdot 62$ & $0 \cdot 294$ & $2 \cdot 2$ \\
\hline Bottled sparkling water & 93 & 276 & $33 \cdot 7$ & 41 & 152 & $27 \cdot 0$ & $1 \cdot 25$ & $0 \cdot 92-1 \cdot 70$ & $0 \cdot 158$ & $68 \cdot 9$ \\
\hline Tap water & 12 & 31 & $38 \cdot 7$ & 121 & 397 & $30 \cdot 5$ & $1 \cdot 27$ & $0 \cdot 80-2 \cdot 03$ & $0 \cdot 420$ & 8.9 \\
\hline Beer & 34 & 121 & $28 \cdot 1$ & 100 & 306 & $32 \cdot 7$ & $0 \cdot 86$ & $0 \cdot 62-1 \cdot 19$ & $0 \cdot 418$ & $25 \cdot 2$ \\
\hline Dry cake & 56 & 144 & $38 \cdot 9$ & 68 & 255 & $26 \cdot 7$ & $1 \cdot 46$ & $1 \cdot 09-1 \cdot 95$ & $0 \cdot 013$ & $41 \cdot 5$ \\
\hline Bread & 101 & 276 & $36 \cdot 6$ & 24 & 137 & $17 \cdot 5$ & $2 \cdot 09$ & $1 \cdot 41-3 \cdot 10$ & $<0.000$ & $74 \cdot 8$ \\
\hline Butter & 82 & 236 & $34 \cdot 8$ & 43 & 172 & $25 \cdot 0$ & $1 \cdot 39$ & $1 \cdot 02-1 \cdot 90$ & 0.039 & $60 \cdot 7$ \\
\hline Coffee & 88 & 266 & $33 \cdot 1$ & 44 & 156 & $28 \cdot 2$ & $1 \cdot 17$ & $0 \cdot 87-1 \cdot 59$ & $0 \cdot 328$ & $65 \cdot 2$ \\
\hline Tea & 5 & 17 & $29 \cdot 4$ & 129 & 406 & $31 \cdot 8$ & 0.93 & $0.44-1.96$ & $1 \cdot 000$ & $3 \cdot 7$ \\
\hline Milk & 34 & 93 & $36 \cdot 6$ & 99 & 326 & $30 \cdot 4$ & $1 \cdot 20$ & $0 \cdot 88-1 \cdot 65$ & $0 \cdot 259$ & $25 \cdot 2$ \\
\hline
\end{tabular}

AR, Attack rate; RR, relative risk; CI, confidence interval.

* Fisher's exact $P$ value.

$\dagger$ The salad contained lettuce, tomatoes, cucumber and sweetcorn.

performed (data not shown). Although some of these items were significantly associated with illness, the RRs were $<2$ and none accounted for more than $80 \%$ of cases.

In a multivariable analysis which included three food items (cheese sandwich, salad, mashed potatoes) as well as sex and age group, only the cheese sandwich and the salad continued to show a significant association with illness: the odds ratio (OR) for the cheese sandwich was highest at $6.57(95 \%$ CI $1.53-28 \cdot 28$, $P=0.011)$ while the OR for salad was $2.49(95 \%$ CI $1 \cdot 12-5 \cdot 54, P=0 \cdot 026)$.

\section{Environmental investigation}

General food-handling practices at the hotel were considered to be good; no lapses in temperature control or general hygiene were identified. Further investigation of the cucumber used in the cheese sandwiches revealed that it was supplied by a wholesaler who had washed, sliced and packaged the product in sealed plastic bags prior to delivery. No further washing of the cucumber slices was performed at the hotel. At the same time, the supplier had also delivered whole, unwashed cucumbers which were used in the salad served at lunch time; these cucumbers were washed and chopped at the hotel. It was subsequently established that cucumber slices left over from making the cheese sandwiches had been added to the salad. Further investigations revealed that the pre-sliced cucumbers had been supplied to the wholesaler by an intermediate supplier who had imported them from Spain. The whole cucumbers used in the salad came from a different intermediate supplier and the country of origin could not be traced. 


\section{DISCUSSION}

We describe the first identified foodborne outbreak to be associated with the microsporidian E. bieneusi. Our investigations suggest that cucumber slices in both cheese sandwiches and a salad were the most probable vehicle of transmission. Since no leftover food samples were available for testing and because little is known about $E$. bieneusi in the context of foodborne outbreaks, it is difficult to conclusively implicate this organism as the agent responsible for the outbreak. However, the finding that all six samples available for genotyping were genetically indistinguishable (genotype C) together with the fact that, despite extensive testing, no other organisms were identified in the stool samples strongly suggest that E. bieneusi was the causative agent. Furthermore, the finding that all 19 stool samples from persons belonging to the same professional group who had not attended the event were negative for microsporidia provides additional evidence that the detection of E. bieneusi was not a chance finding. Although these samples were taken 7 months after the event, they nevertheless provide an indication of the prevalence of microsporidia in a population with similar demographic characteristics.

The only outbreak of microsporidia reported in the literature was identified retrospectively by analysis of all samples submitted for microsporidial testing during a 3.5-year period in France; the cause of the outbreak was attributed to drinking water because case-patients lived in the same water distribution area; however, because no analytical epidemiological study was done it is difficult to draw meaningful conclusions from this data [13]. Nevertheless, food- and waterborne routes of transmission of microsporidia are biologically plausible and are supported by several findings including: environmental resistance of spores, probable resistance to disinfectants, survival of spores in water for extended periods, and the detection of spores in ground [21], surface [21, 22], and crop-irrigation [23] waters, and in fresh produce such as raspberries, beans and lettuce [24].

We cannot state with certainty how and where the sliced cucumbers were contaminated. Contamination during final preparation at the hotel seems unlikely because the cucumbers were not processed any further but were added directly to the sandwiches. Furthermore, a high contamination dose is suspected (due to high attack rate in a healthy population) which is unlikely to have occurred because preparation of the sandwiches was carried out by an asymptomatic food handler. The sealed bags of cucumber slices had been refrigerated before use so it is improbable that contamination took place during storage. Similarly, contamination during initial processing at the wholesale supplier, although possible, seems unlikely based on the description of the procedures used. The most likely hypothesis of contamination is that it occurred before harvest, either by contaminated manure, manure compost, sewage sludge, irrigation water, runoff water from livestock operations or directly from wild and domestic animals. These potential contamination events are all plausible and consistent with the assumption that the level of contamination must have been high. Unfortunately, because we were unable to trace the cucumbers back to the farm where they were grown, we could not investigate these possible contamination routes further. However, additional information is provided by the genotyping results. While there have been several cases of genotype $\mathrm{C}$ identified in humans, predominantly in HIV-negative organ transplant recipients in Europe $[25,26]$, there is only one report on animals in the literature [27]. Thus, while a zoonotic link cannot be ruled out, the involvement of this genotype suggests that the source of contamination in this outbreak was of human (faecal) origin.

While thorough washing of fresh produce remains of utmost importance in preventing foodborne illness and should continue to be emphasized, sometimes washing may be insufficient to remove all pathogens. In this instance, it may have been that the level of contamination was so high that washing was unable to remove enough of the microbial load so as to prevent infection. Alternatively, it may be that microsporidian spores are capable of strong adhesion to, or internalization in, certain types of produce, thereby successfully evading the effects of washing and disinfection. A recent paper by researchers in the USA demonstrated that Cryptosporidium oocysts were capable of strongly adhering to spinach plants after contact with contaminated water and were also internalized within the leaves, thus making washing entirely ineffective [28].

Several limitations apply to our findings. First, the long incubation period resulted in a substantial delay before the outbreak was recognized and this delayed the start of the epidemiological investigation. As a result, the web-based questionnaire was sent out almost 4 weeks after the event and this may have resulted in recall bias. An additional consequence of 
the delay was that it was not feasible to enquire about the amounts of foods consumed so we were unable to calculate dose response. This is unfortunate because very little is known regarding infection with $E$. bieneusi in healthy populations and we strongly suspect that the high variability in the incubation period, spectrum, severity and duration of symptoms could be explained by dose response. Another limitation associated with the delayed recognition of the outbreak was that only $8 / 95$ initial samples remained for extended analysis. Furthermore, these samples had been fixed in formalin $\geqslant 6$ weeks before DNA extraction and this may have influenced the low PCRpositivity rate (4/8). In contrast, all three stool samples from late November which had been stored unfixed were positive by PCR.

This outbreak provided a unique opportunity to study the incubation period, symptom duration and spectrum of symptoms of E. bieneusi in otherwise healthy persons, which until now was largely unknown. For the first time, we have been able to document incubation periods for cases of microsporidiosis. However, because this was an observational study, it is important to consider the factors that could have influenced the wide range of incubation periods we report here. First, it is plausible that differences in dose response could account for some of the variation because it can be expected that those persons who consumed large amounts of the implicated foods might have had shorter incubation periods. Another factor which might explain the variation is differences in host immunity. Although we believe that our case-patients were immunocompetent persons, we did not systematically collect information about their medical history. We therefore performed a follow-up cohort study to investigate this possibility further (data will be published separately). The lack of specificity in the definition of several of the symptoms might explain some of the variation. Neither the first case-patient nor any of the casepatients with late-onset dates were microbiologically confirmed. The range of incubation periods for the four confirmed case-patients (3-15 days) is therefore more reliable and comparable to other protozoan parasites such as Cryptosporidium and Giardia: 1-12 days and 3-25 days, respectively [29].

Our findings indicate that E. bieneusi is capable of causing an outbreak of gastrointestinal illness in a group of apparently immunocompetent persons. Whether E. bieneusi is newly emerging as a potential agent of foodborne outbreaks or whether it has simply been under-reported previously remains unclear but it is believed that foodborne protozoan infections are almost certainly under-detected by a factor of $\geqslant 10$ [30]. Moreover, the aetiological agent remains unknown for more than half of all reported foodborne outbreaks in Sweden [31]. We hope that by reporting the findings of this investigation, the awareness of considering parasites such as microsporidia as possible agents during outbreaks will be raised, allowing for easier recognition of outbreaks. In particular, we recommend that microsporidia be explored as causative agents in food- and waterborne outbreaks involving long incubation periods $(\geqslant 1$ week), predominantly non-bloody diarrhoea, abdominal pain and bloating, especially when no other organism has been identified.

\section{ACKNOWLEDGEMENTS}

We acknowledge Eva Andersson and Ingrid Persson for their help during the initial investigation and prevalence study. We thank Annika Hulu and Lotta Bertilsson for carrying out the environmental investigation and Yen Ngo and Anna-Maria Kling for statistical support. We thank Thomas Ahlqvist for performing the initial laboratory investigations and Henrik Vedel Nielsen for performing the realtime PCR. Finally, we thank Viviane Bremer and Katharina Alpers (EPIET) for reviewing this manuscript and Margarita Riera-Montes for support during the investigation. The EPIET fellowship of V. Decraene is funded by the European Commission DG SANCO.

\section{DECLARATION OF INTEREST}

None.

\section{REFERENCES}

1. Didier ES. Microsporidiosis: an emerging and opportunistic infection in humans and animals. Acta Tropica 2005; 94: 61-76.

2. Lanternier F, et al. Microsporidiosis in solid organ transplant recipients: two Enterocytozoon bieneusi cases and review. Transplant Infectious Disease 2009; 11: 83-88.

3. Rabodonirina M, et al. Microsporidiosis and transplantation: a retrospective study of 23 cases. Journal of Eukaryotic Microbiology 2003; 50 (Suppl.): 583.

4. Sing A, et al. Molecular diagnosis of an Enterocytozoon bieneusi human genotype $\mathrm{C}$ infection in a moderately 
immunosuppressed human immunodeficiency virus seronegative liver-transplant recipient with severe chronic diarrhea. Journal of Clinical Microbiology 2001; 39: 2371-2372.

5. Fournier S, et al. Microsporidiosis due to Enterocytozoon bieneusi infection as a possible cause of traveller's diarrhea. European Journal of Clinical Microbiology and Infectious Diseases 1998; 17: 743-744.

6. Didier ES, et al. Epidemiology of microsporidiosis: sources and modes of transmission. Veterinary Parasitology 2004; 126 : 145-166.

7. Lores B, et al. Intestinal microsporidiosis due to Enterocytozoon bieneusi in elderly human immunodeficiency virus-negative patients from Vigo, Spain. Clinical Infectious Diseases 2002; 34: 918-921.

8. Wichro E, et al. Microsporidiosis in travel-associated chronic diarrhea in immune-competent patients. American Journal of Tropical Medicine and Hygiene 2005; 73: 285-287.

9. Mathis A, Weber R, Deplazes P. Zoonotic potential of the microsporidia. Clinical Microbiology Reviews 2005; 18: 423-445.

10. Bart A, et al. Frequent occurrence of human-associated microsporidia in fecal droppings of urban pigeons in Amsterdam, the Netherlands. Applied and Environmental Microbiology 2008; 74: 7056-7058.

11. Lobo ML, et al. Identification of potentially humanpathogenic Enterocytozoon bieneusi genotypes in various birds. Applied and Environmental Microbiology 2006; 72: 7380-7382.

12. Santin M, Fayer R. Microsporidiosis: Enterocytozoon bieneusi in domesticated and wild animals. Research in Veterinary Science. Published online: 9 August 2010. doi:10.1016/j.rvsc.2010.07.014.

13. Cotte $\mathbf{L}$, et al. Waterborne outbreak of intestinal microsporidiosis in persons with and without human immunodeficiency virus infection. Journal of Infectious Diseases 1999; 180: 2003-2008.

14. Svedhem V, et al. Disseminated infection with encephalitozoon intestinalis in AIDS patients: report of 2 cases. Scandinavian Journal of Infectious Diseases 2002; 34: 703-705.

15. Svedhem V, et al. Microsporidia in duodenal biopsies from $72 \mathrm{HIV}$-infected patients with abdominal complaints. Acta Pathologica Microbiologica et Immunologica Scandinavica 1998; 106: 535-538.

16. Svenungsson B, et al. Intestinal microsporidiosis in a HIV-seronegative patient. Scandinavian Journal of Infectious Diseases 1998; 30: 314-316.

17. Svenungsson B, et al. Enteropathogens in adult patients with diarrhea and healthy control subjects: a 1-year prospective study in a Swedish clinic for infectious diseases. Clinical Infectious Diseases 2000; 30: 770-778.

18. Weber $\mathbf{R}$, et al. Improved light-microscopical detection of microsporidia spores in stool and duodenal aspirates.
The Enteric Opportunistic Infections Working Group. New England Journal of Medicine 1992; 326: 161-166.

19. Buckholt MA, Lee JH, Tzipori S. Prevalence of Enterocytozoon bieneusi in swine: an 18-month survey at a slaughterhouse in Massachusetts. Applied and Environmental Microbiology 2002; 68: 2595-2599.

20. Verweij JJ, et al. Multiplex detection of Enterocytozoon bieneusi and Encephalitozoon spp. in fecal samples using real-time PCR. Diagnostic Microbiology and Infectious Disease 2007; 57: 163-167.

21. Dowd SE, Gerba CP, Pepper IL. Confirmation of the human-pathogenic microsporidia Enterocytozoon bieneusi, Encephalitozoon intestinalis, and Vittaforma corneae in water. Applied and Environmental Microbiology 1998; 64: 3332-3335.

22. Fournier S, et al. Detection of microsporidia in surface water: a one-year follow-up study. FEMS Immunology and Medical Microbiology 2000; 29: 95-100.

23. Thurston-Enriquez JA, et al. Detection of protozoan parasites and microsporidia in irrigation waters used for crop production. Journal of Food Protection 2002; 65: 378-382.

24. Jedrzejewski S, et al. Quantitative assessment of contamination of fresh food produce of various retail types by human-virulent microsporidian spores. Applied and Environmental Microbiology 2007; 73: 4071-4073.

25. ten Hove RJ, et al. Characterization of genotypes of Enterocytozoon bieneusi in immunosuppressed and immunocompetent patient groups. Journal of Eukaryotic Microbiology 2009; 56: 388-393.

26. Liguory O, et al. Evidence of different Enterocytozoon bieneusi genotypes in patients with and without human immunodeficiency virus infection. Journal of Clinical Microbiology 2001; 39: 2672-2674.

27. Sak B, et al. The first report on natural Enterocytozoon bieneusi and Encephalitozoon spp. infections in wild East-European house mice (Mus musculus musculus) and West-European house mice (M. m. domesticus) in a hybrid zone across the Czech Republic-Germany border. Veterinary Parasitology. Published online: 12 Jan 2011. doi: 10.1016/j.vetpar.2010.12.044

28. Macarisin D, Bauchan G, Fayer R. Spinacia oleracea L. leaf stomata harboring Cryptosporidium parvum oocysts: a potential threat to food safety. Applied and Environmental Microbiology 2010; 76: 555-559.

29. Heymann DL (ed.). Control of Communicable Diseases Manual, 19th edn. Washington D.C.: American Public Health Association, 2008.

30. Casemore DP. Foodborne protozoal infection. Lancet 1990; 336: 1427-1432.

31. Lindblad M, et al. Food poisoning in Sweden: analysis of reported foodborne disease incidents, 2003-2007. National Food Administration, Sweden; Swedish Institute for Infectious Diseases, 2009. Report No. $16-2009$. 